\title{
Instability of a viscous interface under horizontal quasi-periodic oscillation
}

\author{
M. ASSOUL ${ }^{1}$, A. EL JAOUAHIRY ${ }^{1}$, M. ECHCHADLI ${ }^{1}$ and S. ANISS ${ }^{1}$ \\ 1. University of Hassan II, Faculty of Sciences Aïn-Chock, Laboratory of Mechanic, B.P.5366 Maarif, \\ Casablanca, Morocco. mouh.assoul@gmail.com
}

\begin{abstract}
We study the linear stability of two superposed layers of viscous, immiscible fluids of different densities. The whole system is subject to horizontal quasi-periodic oscillation with two incommensurates frequencies $\omega_{1}$ and $\omega_{2}$. The spectral method and Floquet's theory combined with Runge-Kutta method are used to solve numericelly the linear problem. We analyse the influence of the frequencies ratio, $\omega=\frac{\omega_{2}}{\omega_{1}}$, on the mariginal stability. The numerical solution shows that the quasi-periodic excitation has a stabilizing or a destabilizing effect on the KelvinHelmholtz instability as well as in the parametric resonances depending on the frequency ratio and the amplitudes ratio $\alpha=\frac{a_{2}}{a_{1}}$.
\end{abstract}

Keywords Linear stability; quasi-periodic oscillation; Runge-Kutta; Floquet's theory; instability of KelvinHelmholtz; parametric resonance.

\section{Introduction}

Several works have been carried out to study the interfacial instability of two superposed layers subject to periodic oscillations. Khenner and al. [1], have studied the linear stability of the interface between two fluids filling a cavity which performs horizontal harmonic oscillations. They have reduced the linear stability problem, using the inviscid approximation to the Mathieu equation. Hereafter, they have examined the parametric resonant regions of instability associated with the intensification of the capillary waves at the interface and they have compared their results to those found in the viscous case in a fully numerical investigation.

Recently, the linear stability analysis model carried out by Khenner and $a l$. [1] has been extended both theoretically and experimentally by Talib and al. [2] to include the effect of the viscosities of the fluid layers of finite depth and confined between horizontal boundaries. In these studies, the authors have solved numerically, using spectral methods, the linear stability problem for an exhaustive range of vibrational to viscous force ratios and viscosity contrasts. They have shown that the viscous model allows to predict the onset of each mode of instability, particularly in the limit of high-viscosity contrast. Talib and al. [2] have characterized the evolution of neutral curves from the multiple modes of the parametric resonant instability to the single-frozen-wave mode encountered in the limit of practical flows. They have found that the interface is linearly unstable to a Kelvin-Helmholtz mode and to successive parametric-resonance modes.

In this work, we extend the study in reference [2] by considering quasi-periodic oscillations with two incommensurate frequencies $\omega_{1}$ and $\omega_{2}$. We focuse attention on the influence of the frequency ratio and the amplitudes of accelerations of the oscillatory motion, $a_{1} \omega_{1}^{2}$ and $a_{2} \omega_{2}^{2}$, on the marginal stability in terms of amplitude, $A=\frac{a_{1}}{d_{2}}$, as a function of the wave number.

\section{2 formulation}

We consider two superposed layers of immiscible and incompressible Newtonian fluids, having two kinematic viscosities $\nu_{1}$, and $\nu_{2}$, densities $\rho_{1}$ and $\rho_{2}$ and which are bounded below and above by rigid boundaries (see Figure 1). The dense fluid occupies the lower region of height $d_{1}$ and the least dense fluid occupies the upper region of height $d_{2}$. The system is subjected to an oscillating motion according to the law: $\rho_{\beta} a_{1} \omega_{1}^{2} \cos \left(\omega_{1} t^{*}\right)+\rho_{\beta} a_{2} \omega_{2}^{2} \cos \left(\omega_{2} t^{*}\right)$ and to the force of gravity $\rho_{\beta} \mathbf{g}(\beta=1,2)$, where $a_{1}, a_{2}$, $\omega_{1}$ and $\omega_{2}$ denote respectively the displacement amplitudes and frequencies of the oscillatory motion. The interfacial tension between the fluids is denoted by $\gamma$.

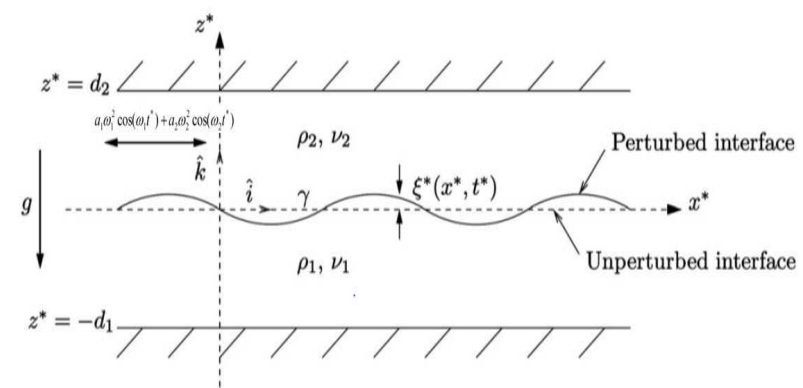

FIG. 1. Schematic diagram of the two-layer fluid system in the frame of reference of the oscillating rigid boundaries.

Using $d_{2}, a_{1} \omega_{1}, \omega_{1}^{-1}, \rho_{2} v_{2} a_{1} \omega_{1} / d_{2}$ as length, velocity, time, and pressure scales, respectively, the dimensionless equations governing the two-layer flows, in the relative frame, are:

$$
\Omega \frac{\partial \mathbf{V}_{\beta}}{\partial t}+A \Omega\left(\mathbf{V}_{\beta} \nabla\right) \mathbf{V}_{\beta}=-R_{\beta} \nabla P_{\beta}-\frac{G_{0} \Omega}{A} \mathbf{k}
$$




$$
\begin{gathered}
+\frac{1}{N_{\beta}} \Delta \mathbf{V}_{\beta}+\Omega\left(\cos (t)+\alpha \omega^{2} \cos (q t)\right) \mathbf{i} \\
\nabla \cdot \mathbf{V}_{\beta}=0
\end{gathered}
$$

where $\mathbf{V}_{\beta}=\left(U_{\beta}, W_{\beta}\right)$ is the velocity in each fluid layer, $\Omega=\left(\omega_{1} d_{2}^{2} / v_{2}\right)$ and $A=a_{1} / d_{2}$ are the dimensionless frequency and amplitude of oscillations, respectively, $R_{\beta}=\rho_{2} / \rho_{\beta}$ and $N_{\beta}=v_{2} / v_{\beta}$ where $R_{2}=N_{2}=1$ are the ratios of densities and viscosities, respectively, $\omega=\omega_{2} / \omega_{1}$ and $\alpha=a_{2} / a_{1}$ are the ratio of frequencies and amplitudes of oscillation, respectively, and $G_{0}=$ $g /\left(d_{2} \omega_{1}^{2}\right)=A^{2} F r^{-1}$ where $F r=\left(a \omega_{1}\right)^{2} / g d_{2}$ is a modified inverse Froude number. The no-slip conditions and the boundary conditions at the interface $\xi(x, t)$ are given by:

$$
\begin{gathered}
\mathbf{V}_{\mathbf{1}}=0 \quad \text { en } \quad z=-d, \\
\mathbf{V}_{\mathbf{1}}=0 \quad \text { en } \quad z=1, \\
\frac{1}{A} \frac{\partial \xi}{\partial t}+\left(\mathbf{V}_{\mathbf{1}} \nabla\right) \xi=\mathbf{V}_{\mathbf{1}} \cdot \mathbf{k}, \\
\mathbf{V}_{\mathbf{1}}=\mathbf{V}_{\mathbf{1}}, \\
\left(\mathbf{n} \cdot \pi_{1}\right) \cdot \mathbf{n}-\left(\mathbf{n} \cdot \pi_{2}\right) \cdot \mathbf{n}=\frac{\Omega}{W_{e} A} \nabla \cdot \mathbf{n}, \\
\left(\mathbf{t} \cdot \pi_{1}\right) \cdot \mathbf{n}=\left(\mathbf{t} \cdot \pi_{2}\right) \cdot \mathbf{n}, \\
\int_{-d}^{\xi} \mathbf{V}_{\mathbf{1}} \cdot \mathbf{i} d z+\int_{\xi}^{1} \mathbf{V}_{\mathbf{1}} \cdot \mathbf{i} d z=0 .
\end{gathered}
$$

where $d=\frac{d_{1}}{d_{2}}, \pi_{\beta}=-P_{\beta} I+\frac{1}{R_{\beta} N_{\beta}}\left[\nabla \mathbf{V}_{\beta}+\left(\nabla \mathbf{V}_{\beta}\right)^{T}\right]$ is the stress tensor in each fluid and $W_{e}=\rho_{2} d_{2}^{3} \omega^{2} / \gamma$ is the Weber number, $\mathbf{n}$ is the outward normal unit vector pointing from fluid 1 into fluid 2 and $\mathbf{t}$ is the tangent unit vector on the interface.

\section{Base flow solution}

The base flow, $\left(\bar{V}_{\beta}, \bar{P}_{\beta}\right)$, is quasi-periodic, parallel to the horizontal boundaries and the interface remains unperturbed. It takes the form:

$$
\begin{gathered}
\bar{U}_{\beta}(z, t)=\Re\left[\Phi_{1 \beta}(z) \cdot e^{i t}+\Phi_{2 \beta}(z) \cdot e^{i \omega t}\right] \\
\bar{P}_{\beta}=\Re\left[-\frac{G_{0} \Omega}{R_{\beta} A} z+x\left(S_{\beta} e^{i t}+F_{\beta} e^{i \omega t}\right)+C\right]
\end{gathered}
$$

where:

$$
\begin{aligned}
& \Phi_{1 \beta}(z)=A_{1 \beta} e^{m_{\beta} z}+B_{1 \beta} e^{-m_{\beta} z}+i\left(\frac{R_{\beta} S}{\Omega}-1\right) \\
& \Phi_{2 \beta}(z)=A_{2 \beta} e^{w_{\beta} z}+B_{2 \beta} e^{-w_{\beta} z}+i\left(\frac{R_{\beta} F}{\omega \Omega}-\alpha \omega\right) . \\
& m_{\beta}=\sqrt{i \Omega N_{\beta}} \\
& w_{\beta}=\sqrt{i \Omega N_{\beta} \omega}
\end{aligned}
$$

The integration constants $A_{1 \beta}, A_{2 \beta}, B_{1 \beta}, B_{2 \beta}, F$ and $S$ are determined by imposing conditions $(3),(4),(6),(8)$ and (9) and $C$ is an arbitrary constant.
Note that for $\alpha=0$, periodic case, we get the same profiles of velocity obtained by Talib and $a l$. [2].

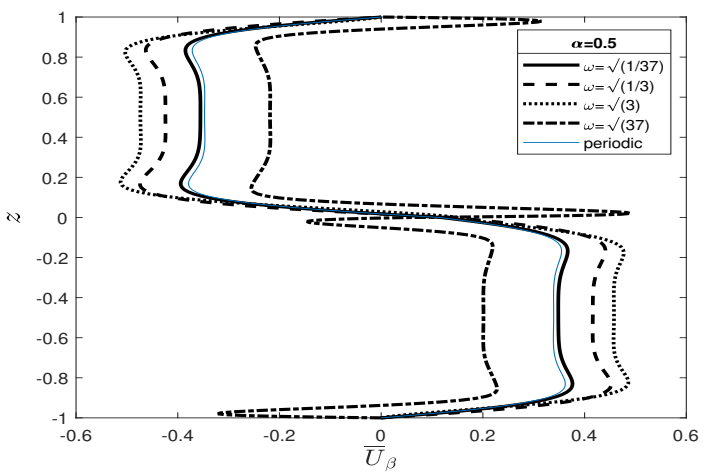

FIG. 2. Base flow solution at $t=\pi / 2$, calculated for $R_{1}=0.49, d=1, N_{1}=1, \Omega=3.927 \times 10^{2}$ and for different ratios of frequency.

\section{Perturbation equations}

We derive the stability equations by expressing the governing equations (1) ans (2) in terms of the stream function, $\psi_{\beta}$, defined as

$$
u_{\beta}=\frac{\partial \psi_{\beta}}{\partial z} \quad \text { and } \quad w_{\beta}=-\frac{\partial \psi_{\beta}}{\partial x}
$$

The small normal perturbation of stream function $\psi_{\beta}$ and the interface position $\xi$ are searched in the following form:

$$
\left(\psi_{\beta}, \xi\right)=\left(\bar{\psi}_{\beta}, 0\right)+\left[\varphi_{\beta}(z, t), h(t)\right] e^{i k x}+c . c .,
$$

where $k=2 \pi d_{2} / \lambda^{*}$ is the dimensionless wavenumber of the disturbance, $\lambda^{*}$ is the dimensional wavelength, and c.c. denotes the complex conjugate. By substituting (12) into the Navier-Stokes equations, as well as the boundary and interfacial conditions, subtractiong out the base state, eliminationg the pressure term, and neglecting higher order terms, we obtain the following Orr-sommerfeld equations for the two layer flows:

$$
\begin{aligned}
& \left(\Omega \frac{\partial}{\partial t}+i k A \Omega \bar{U}_{\beta}\right)\left(\phi_{\beta}^{\prime \prime}-k^{2} \phi_{\beta}\right)-i k A \Omega U_{\beta}^{\prime \prime} \phi_{\beta} \\
& +\frac{1}{N_{\beta}}\left(2 k^{2} \phi_{\beta}^{\prime \prime}-k^{4} \phi_{\beta}-\phi_{\beta}^{\prime \prime \prime \prime}\right)=0
\end{aligned}
$$

where the prime $\left({ }^{\prime}\right)$ denotes partial differentiation with respect to $z$. The no-slip boundary conditions become:

$$
\phi_{1}=\phi_{1}^{\prime}=0 \text { en } z=-d, \phi_{2}=\phi_{2}^{\prime}=0 \text { en } z=1
$$

The linearized interface conditions, applied at $z=0$, become:

$$
\begin{gathered}
\frac{1}{A} \frac{\partial h}{d t}+i k \bar{U}_{1} h+i k \phi_{1}=0 \\
\phi_{1}^{\prime}-\phi_{2}^{\prime}+h\left(\bar{U}_{1}^{\prime}-\bar{U}_{1}^{\prime}\right)=0
\end{gathered}
$$




$$
\begin{gathered}
\phi_{1}-\phi_{2}=0 \\
{\left[\frac{\Omega}{R_{1}} \frac{\partial \phi^{\prime}}{d t}-\frac{i k A \Omega}{R_{1}} \bar{U}_{1}^{\prime} \phi_{1}+\frac{i k A \Omega}{R_{1}} \bar{U}_{1} \phi^{\prime}+\frac{3 k^{2}}{N_{1} R_{1}} \phi_{1}^{\prime}\right.} \\
\left.-\frac{1}{N_{1} R_{1}} \phi^{\prime \prime \prime \prime}{ }_{1}+\frac{i k G_{0} \Omega}{A R_{1}} h\right]-\left[\Omega \frac{\partial \phi^{\prime}}{d t}-i k A \Omega \bar{U}_{2}^{\prime} \phi_{2}\right. \\
\left.+i k A \Omega \bar{U}_{2} \phi^{\prime}{ }_{2}+3 k^{2} \phi^{\prime}{ }_{2}-\phi^{\prime \prime \prime}{ }_{2}+\frac{i k G_{0} \Omega}{A} h\right] \\
+\frac{i k^{3} \Omega}{\hat{W}_{e} A} h=0 \\
\phi_{1}^{\prime \prime}+h \bar{U}_{1}^{\prime \prime}+k^{2} \phi_{1}-N_{1} R_{1}\left(\phi^{\prime \prime}{ }_{2}+h \bar{U}_{2}^{\prime \prime}+k^{2} \phi_{2}\right)=0
\end{gathered}
$$

\section{Numerical solution}

To solve equations (13)-(19), we use the spectral methods based on the Gausse-Lobatto collocation points for spatial resolution [3, 4]. The chebyshev polynomials are defined in the meantime $[-1,1]$. In order to achieve high resolution near the boundaries and the interface, the lower and upper layers are mapped onto Chebyshev space, $\zeta \in[-1,1]$, with the transformations $\zeta=\frac{2 z+d}{d}$ and $\zeta=1-2 z$, respectively, so that the interface is placed at $\zeta=1$. One uses the fact that an irrational number, $\omega=\frac{\omega_{2}}{\omega_{1}}$, can be approximated by a rational number of the form $\omega=\frac{p}{q}$, where $p$ and $q$ are relatively prime integers. Finally we use the Floquet's theory combined with the 4th order Runge-Kutta method for temporal resolution.

\section{Results and conclusion}

We determine the value of the amplitude, A, at which the parallel shear flow becomes unstable to standing waves of wavenumber $\mathrm{k}$. The neutral stability curves are shown in Figures 3 and 4, for different values of the irrational ratio of frequencies $\omega$.

We assume in figure 3 that the amplitudes of acceleration oscillatory motion are equal, i.e., $a_{1} \omega_{1}^{2}=a_{2} \omega_{2}^{2}$. In this case we find that the increasing of the $\omega$ has a stabilizing effect, also for the hight values of $\omega$, the marginal stability curves converge toward the stability boundaries of the periodic case that was developped by Khennner [1] and Talib [2]. Note that the Kelvin-Helmholtz region is increasing when the $\omega$ is increasing.

Figure 4 shows the effect of $\omega$ when the amplitudes ratio, $\alpha$, is assigned. The increase of $\omega$ destabilizes the limit of small values of $k$ more strongly than the resonant mode at higher $k$. Also, for the low frequency ratios, $\omega_{1} \gg \omega_{2}$, the neutral curve converge to the case of periodic oscillation.

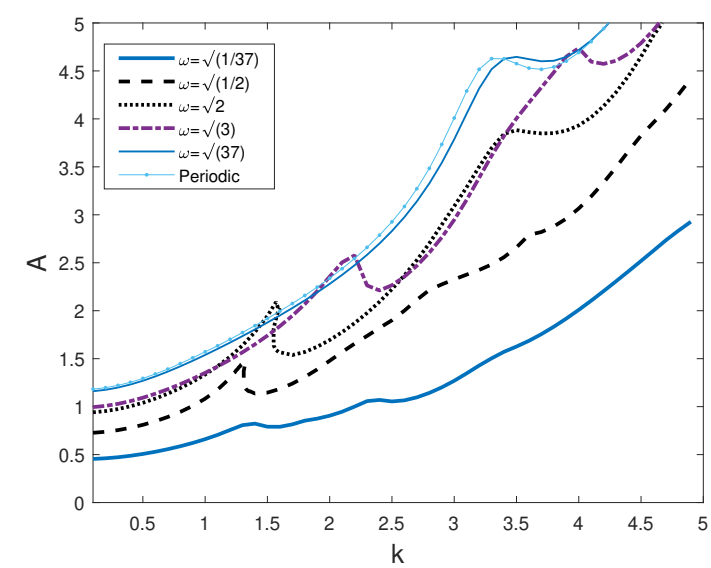

FIG. 3. Marginal stability curves showing the amplitude, $A$, against the wavenumber, $k$ for $\Omega=25$ and for $\alpha \omega^{2}=1$. The other parameter values are $R_{1}=0.5$, $N_{1}=1, G_{0}=0.16, W_{e}=6.25$ and $d=1$.

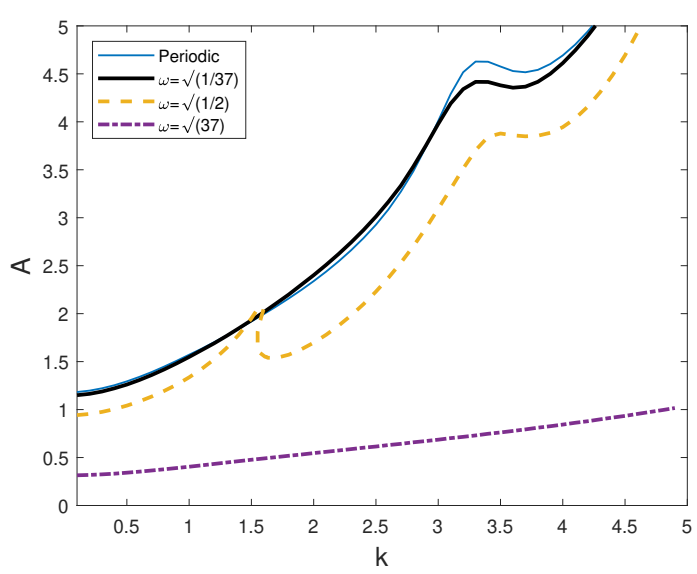

FIG. 4. Marginal stability curves showing amplitude, A, against the wavenumber, $\mathrm{k}$ for $\Omega=25, \alpha=0.5$ and for different values of the frequency ratio. The other parameter values are $R_{1}=0.5, N_{1}=1, G_{0}=0.16, W_{e}=6.25$ and $d=1$.

\section{References}

[1] M. V. Khenner, D. V. Lyubimov, T. S. Belozerova, and B. Roux, Stability of plane-parallel vibrational flow in a two-layer system, Eur. J. Mech. B/Fluids 18, (1999), 1085.

[2] E. Talib and A. Juel, Instability of a viscous interface under horizontal oscillation, Phys. Fluids 19, (2007), 092102.

[3] L. N. Trefethen, Spectral Methods in Matlab, SIAM, Philadelphia, 2000.

[4] A. C. Weideman, S. C. Reddy, A MATLAB differentiation matrix suite, ACM Trans. Math. Softw, 26, 465-519, 2000. 